\title{
TOPOLOGICAL ESSENTIALITY AND NONLINEAR BOUNDARY VALUE CONTROL PROBLEMS*
}

\author{
LeCh GóRniewicz - PAOLO Nistri
}

\begin{abstract}
A concept of topological essentiality is used to prove several existence results for nonlinear boundary value control problems. Some examples to illustrate the obtained results are presented.
\end{abstract}

\section{Introduction}

The aim of this paper is to study boundary value problems for the nonlinear control system of the following form

$$
\dot{x}(t)=f(t, x(t), u(t)),
$$

where $f:[0,1] \times \mathbb{R}^{n} \times \mathbb{R}^{m} \rightarrow \mathbb{R}^{n}$ is a continuous function or, more generally, it satisfies some Carathéodory-type conditions that will be specified later.

Moreover, we will deal with boundary value problems for the $k$-order control problem of the form

$$
x^{(k)}(t)=f\left(t, x(t), \dot{x}(t), \ldots, x^{(k-1)}(t), u(t)\right)
$$

with $f:[0,1] \times \mathbb{R}^{n k} \times \mathbb{R}^{m} \rightarrow \mathbb{R}^{n}$ as before.

1991 Mathematics Subject Classification. 34H05, 34B15, 93B05.

Key words and phrases. Essential maps, nonlinear control systems, boundary value problems.

* Research supported in part by CNR (Italy) and by research project MURST 'Teoria del controllo dei sistemi dinamici' and by Polish KBN grant. 
In order to define what we mean by a solution of a nonlinear boundary value control problem associated to $(0.1)$ or to $(0.2)$ we will introduce some notations.

We will consider several function spaces. By a normed (Banach) space we always understand a real normed (Banach) space. We let

(0.3) $L_{1}=L_{1}\left([0,1], \mathbb{R}^{n}\right)$ - the Banach space of (Lebesque) integrable functions,

(0.4) $C^{k}=C^{k}\left([0,1], \mathbb{R}^{n}\right)$ - the Banach space of $C^{k}$-functions, $k \geq 0$,

(0.5) $A C=A C\left([0,1], \mathbb{R}^{n}\right)$ - the Banach space of absolutely continuous function equipped with the norm defined as follows

$$
\|x\|_{A C}=|x(0)|+\int_{0}^{1}|\dot{x}(t)| d t,
$$

(0.6) if $E, F$ are two normed (Banach) spaces then the Cartesian product $E \times F$ is equipped with the following norm

$$
\|(x, u)\|_{E \times F}=\max \left\{\|x\|_{E},\|u\|_{F}\right\} .
$$

We reserve the notation $|\cdot|$ for the norm in finite dimensional spaces. By a solution of a nonlinear boundary value control problem associated to (0.1) we mean a pair $(x, u) \in A C \times L_{1}$ such that for almost all (a.a.) $t \in[0,1]$ we have

$$
\dot{x}(t)=f(t, x(t), u(t))
$$

and satisfying the assigned boundary conditions.

While by a solution of a nonlinear boundary value control problem associated to $(0.2)$ we mean a pair $(x, u) \in C^{k-1} \times L_{1}$ such that $x^{(k-1)} \in A C$ and such that (0.2) holds true for a.a. $t \in[0,1]$ together with the assigned boundary conditions. Through the paper we will assume that all the solutions are prolongable to $[0,1]$.

Topological methods have been widely employed in the study of control problems. Indeed, fixed point methods both for perturbed linear control systems and fully nonlinear control systems in finite dimension are the main tools to establish controllability in the following papers [9]-[17], [27]-[29], [31] and [39]-[41]. Topological degree theory and related topics are employed in [20], [21], [32] and [33] to treat the same problem.

Fixed point theorems and topological degree theory have been used also for the analysis of infinite dimensional control systems we cite here among many others the following papers [1], [2], [7], [8], [18], [26], [30] and [38].

The multivalued analysis and the related topological tools, mainly: existence selection theory, fixed-point theorems and degree theory, are also of great help in dealing with control and optimization problems both for finite and infinite dimensional control systems. We only mention here the monographs [5], [6] and the papers: [3], [4], [19], [23], [24] and [34]-[36]. 
The aim of this paper is to introduce a different and simpler topological tool for the study of very general nonlinear boundary value control problems. Specifically, we will use the so-called topological essentiality and its existence and homotopy properties [25], [37] to prove the solvability of (0.1) and (0.2) under general boundary conditions involving both the state $x$ and the control $u$.

The paper is organized as follows. In Section 1 we introduce all the relevant background concerning the topological essentiality for admissible multivalued maps. Section 2 is devoted to the control problems. It is divided in three parts, in the first two we consider equation (0.1) under different growth conditions on the dynamics $f$, while in the last part we deal with $(0.2)$.

\section{Multivalued essential maps}

All the considered topological spaces are assumed to be metric. A space $X$ is called an absolute retract (written $X \in \mathrm{AR}$ ) if for each space $Y$ and for any homeomorphism $h: X \rightarrow Y$ such that $h(X)$ is a closed subset of $Y$, the set $h(X)$ is a retract of $Y$; that is, there exists a continuous map $r: Y \rightarrow h(X)$ such that $r(y)=y$ for every $y \in h(X)$.

Note, that any retract of a normed space $E$ is an absolute retract (see [22]). We will consider the Čech homology functor $H$ with compact carriers and rational coefficients $Q$ as defined in [22]. A nonempty compact space $X$ is called acyclic provided

$$
H_{n}(X)= \begin{cases}0 & \text { if } n>0 \\ Q & \text { if } n=0\end{cases}
$$

Let us remark that any contractible space $X$ or in particular, any AR-space $X$ is acyclic. We need also the notion of a Vietoris map. A continuous map $p: \Gamma \rightarrow X$ is called a Vietoris map provided the following two conditions are satisfied.

(i) for each $x \in X$ the set $p^{-1}(x)$ is acyclic;

(ii) $p$ is a proper map, i.e., for every compact $K \subset X$ the set $p^{-1}(K)$ is compact.

In what follows the symbol $p: \Gamma \Rightarrow X$ is reserved for Vietoris mappings.

Let $X$ and $Y$ be two spaces and assume that for any $x \in X$ a nonempty compact subset $\varphi(x)$ of $Y$ is given; in such a case we say that $\varphi: X \multimap Y$ is a multivalued map. The symbol $f: X \rightarrow Y$ is used only for singlevalued maps.

A multivalued map $\varphi: X \multimap Y$ is called upper semicontinuous (u.s.c.) or respectively lower semicontinuous (l.s.c.) provided for any open $V \subset Y$ the set

$$
\varphi^{-1}(V)=\{x \in X \mid \varphi(x) \subset V\}
$$

or the set

$$
\varphi_{+}^{-1}(V)=\{x \in X \mid \varphi(x) \cap V \neq \emptyset\},
$$

respectively, is open. For relevant properties of u.s.c. and l.s.c. mappings, see [22]. 
Definition 1.1 (see [22] and [25]). A multivalued mapping $\varphi: X \multimap Y$ is called admissible provided there is a diagram

$$
X \stackrel{p}{\Longleftarrow} \Gamma \stackrel{q}{\longleftrightarrow} Y
$$

with $q$ continuous map such that $\varphi(x)=q\left(p^{-1}(x)\right)$ for any $x \in X$. Then $(p, q)$ is called a selected pair for $\varphi$ and we write $(p, q) \subset \varphi$.

Since $p$ is proper it follows that any admissible map $\varphi$ is u.s.c. Moreover one can say that values $\varphi(x)$ of any admissible map $\varphi$ are compact, connected and nonempty. An admissible map $\varphi$ is called compact, if there exists a selected pair $(p, q) \subset \varphi$ such that $q$ is a compact map, i.e. $\overline{q(\Gamma)}$ is a compact set.

The following properties are straightforward (see [22]).

Proposition 1.2.

(1.2.1) if $\varphi: X \multimap Y$ is an u.s.c. map with acyclic values, then $\varphi$ is admissible;

(1.2.2) if $\varphi_{1}: X \multimap Y$ and $\varphi_{2}: Y_{1} \multimap Z$ are admissible then so is the composition $\varphi_{2} \circ \varphi_{1}: X \multimap Z$ (with $Y=Y_{1}$ ) and the Cartesian product $\varphi_{1} \times \varphi_{2}: X \times Y_{1} \multimap Y \times Z$.

Some algebraic properties of admissible mappings are considered below.

Proposition 1.3 (see [22] and [25]). Let $E$ be a normed space and $\varphi_{1}, \varphi_{2}$ : $X \multimap E$ two admissible mappings and let $s: E \rightarrow \mathbb{R}$ be a continuous function. Then all the mappings defined below are admissible

(1.3.1) $\left(\varphi_{1}+\varphi_{2}\right): X \multimap E, \quad\left(\varphi_{1}+\varphi_{2}\right)(x)=\left\{u+v \mid u \in \varphi_{1}(x)\right.$ and $\left.v \in \varphi_{2}(x)\right\}$;

(1.3.2) $\left(\varphi_{1}-\varphi_{2}\right): X \multimap E, \quad\left(\varphi_{1}-\varphi_{2}\right)(x)=\left\{u-v \mid u \in \varphi_{1}(x)\right.$ and $\left.v \in \varphi_{2}(x)\right\}$;

(1.3.3) $(s \varphi): X \multimap E, \quad(s \varphi)(x)=\{s(x) u \mid u \in \varphi(x)\}$.

We would like to remark also that the Schauder Fixed Point Theory remains true for admissible mappings.

Theorem 1.4 (Schauder Fixed Point Theorem). If $X \in \mathrm{AR}$ and $\varphi: X \multimap Y$ is an admissible compact map, then there exists $x \in X$ such that $x \in \varphi(x)$.

Now, we are in a position to define the notion of topological essentiality (see [25]) for admissible mappings which we will use in the study of boundary value control problems considered in the next section.

In what follows $E, F$ are two real normed spaces and $U$ is an open bounded subset of $E$. By $\partial U$ we will denote the boundary of $U$ in $E$ and by $\bar{U}$ the closure of $U$ in $E$. We let

$$
\begin{aligned}
A_{\partial U}(U, F) & =\{\varphi: \bar{U} \multimap F \mid \varphi \text { is admissible and } 0 \notin \varphi(\partial U)\} ; \\
A^{C}(U, F) & =\{\varphi: \bar{U} \multimap F \mid \varphi \text { is admissible and compact }\} ; \\
A^{0}(U, F) & =\left\{\varphi: \bar{U} \multimap F \mid \varphi \in A^{C}(U, F) \text { and } \varphi(x)=0 \text { for any } x \in \partial U\right\} .
\end{aligned}
$$


Definition 1.5 (see [25], [37]). A map $\varphi \in A_{\partial U}(U, F)$ is called essential (with respect to $A^{0}(U, F)$ ) provided for any $\psi \in A^{0}(U, F)$ there exists a point $x \in U$ such that $\varphi(x) \cap \psi(x) \neq \emptyset$, i.e. $\varphi$ and $\psi$ have a coincidence in $U$.

Taking $E=F$ the notion of essentiality can be reinterpreted as $Z_{2}$ topological degree. Let us enumerate the most important properties of the above defined topological essentiality.

Proposition 1.6 (see [25]).

(1.6.1) (Existence) If $\varphi$ is essential, then there exists a point $x \in U$ such that $0 \in \varphi(x)$.

(1.6.2) (Compact perturbation) If $\varphi$ is essential and $\eta \in A^{0}(U, F)$, then $(\varphi+\eta)$ is essential.

(1.6.3) (Coincidence) Assume $\varphi$ is essential and $\eta \in A^{C}(U, F)$ is such that the set

$$
A=\{x \in \bar{U} \mid \varphi(x) \cap(t \eta(x)) \neq \emptyset \text { for some } t \in[0,1]\}
$$

is contained in $U$, then $\varphi$ and $\eta$ have a coincidence.

(1.6.4) (Localisation) Let $\varphi \in A_{\partial U}(U, F)$ be essential. Assume that $V$ is an open subset of $U$ such that $\varphi_{+}^{-1}(\{0\}) \subset V$ and $\bar{V} \in \mathrm{AR}$. Then the restriction $\widetilde{\varphi}$ of $\varphi$ to $\bar{V}$ is an essential map.

(1.6.5) (Homotopy) Let $\varphi \in A_{\partial U}(U, F)$ be essential. If $H: \bar{U} \times[0,1] \multimap F$ is a compact admissible map such that

(i) $H(x, 0)=\{0\}$ for any $x \in \partial U$;

(ii) if $\{x \in \bar{U} \mid \varphi(x) \cap H(x, \mu) \neq \emptyset$ for some $\mu \in[0,1]\} \subset U$ then the map $(\varphi-H(\cdot, 1)): \bar{U} \multimap F$ is essential.

(1.6.7) (Essentiality of linear isomorphisms) Let $L: E \rightarrow F$ be a continuous linear isomorphism. Then for any open bounded neighbourhood $U$ of the origin in $E$ the restriction $\widetilde{L}: \bar{U} \rightarrow F$ of $L$ to $\bar{U}$ is essential.

(1.6.8) (Essentiality of homeomorphisms) Let $U \subset E$ be an open bounded set such that $\bar{U} \in \mathrm{AR}$. Assume that $f: \bar{U} \rightarrow F$ is a homeomorphism onto the closed subset $f(\bar{U})$ of $F$ and $f(U)$ is an open subset of $F$ such that $0 \in f(U)$. Then $f$ is essential.

(1.6.9) (Essentiality of Vietoris mappings) If $p: \bar{U} \Rightarrow F$ is a Vietoris map and $p^{-1}\{(0)\} \subset U$ then $p$ is essential.

\section{Topological essentaility and control problems}

In this section we study very general boundary value control problems by means of topological essentiality for multivalued maps. Through this section by $V$ we will denote a finite dimensional subspace of $L_{1}$. For the sake of clarity of the presentation we will divide the section into three parts. Specifically, in 
the first two parts we consider different growth conditions on $f$, namely a fully nonlinear control system and a perturbed linear control system respectively. In the third part we treat higher order control system.

2.1. Nonlinear control systems. Let $f:[0,1] \times \mathbb{R}^{n} \times \mathbb{R}^{m} \rightarrow \mathbb{R}^{n}$ be a mapping satisfying the following conditions.

(2.1.1) $f(t, \cdot, \cdot)$ is continuous for a.a. $t \in[0,1]$;

(2.1.2) $f(\cdot, x, u)$ is (Lebesque) measurable for any $x \in \mathbb{R}^{n}$ and $u \in \mathbb{R}^{m}$;

(2.1.3) for any $\rho>0$ there exists $\alpha_{\rho} \in L_{1}$ such that

$$
|f(t, x, u)| \leq \alpha_{\rho}(t)
$$

for a.a. $t \in[0,1]$ and any $(x, u) \in \mathbb{R}^{n} \times \mathbb{R}^{m}$ such that $|x|+|u| \leq \rho$.

Moreover, assume that

(2.1.4) $l_{i}: A C \times V \rightarrow \mathbb{R}^{n}, i=1, \ldots, r$, is a continuous function;

(2.1.5) $\psi_{i}: A C \times V \multimap \mathbb{R}^{n}, i=1, \ldots, r$, is an admissible and completely continuous map, (i.e., for any bounded set $B \subset A C \times V$ the set $\overline{\psi_{i}(B)}$ is compact).

We will consider the following boundary value problem

$$
\left\{\begin{array}{l}
\dot{x}(t)=f(t, x(t), u(t)), \\
l_{i}(x, u) \in \psi_{i}(x, u), \quad i=1, \ldots, r, \\
x(0) \in \psi_{r}(x, u) .
\end{array}\right.
$$

Let us denote by $S\left(f, l_{i}, \psi_{i}\right)$ the set of all the solution pairs $(x, u)$ of (2.1.6). We will proceed as in the Poincaré continuation method. Therefore for any $\mu \in[0,1]$ we will consider the following problem.

$$
\left\{\begin{array}{l}
\dot{x}(t)=\mu f(t, x(t), u(t)), \\
l_{i}(x, u) \in \mu \psi_{i}(x, u), \quad i=1, \ldots, r, \\
x(0) \in \mu \psi_{r}(x, u) .
\end{array}\right.
$$

We denote by $S_{\mu}\left(f, l_{i}, \psi_{i}\right)$ the set of all solutions of $(2.1 .6)_{\mu}$. Of course $S\left(f, l_{i}, \psi_{i}\right)$ $=S_{1}\left(f, l_{i}, \psi_{i}\right)$. We assume that

(2.1.7) there exists $M>0$ such that for any $\mu \in[0,1]$ and any $(x, u) \in$ $S_{\mu}\left(f, l_{i}, \psi_{i}\right)$ we have

$$
\|(x, u)\|<M .
$$

Now, we associate with (2.1.6) the following map

$$
\left\{\begin{array}{l}
g: A C \times V \rightarrow A C \times \mathbb{R}^{n r}, \\
g(x, u)=\left(x, l_{r}(x, u), l_{r-1}(x, u), \ldots, l_{1}(x, u)\right) .
\end{array}\right.
$$

We can prove the following result. 
THEOREM 2.1.9. Assume that conditions (2.1.1)-(2.1.5) and (2.1.7) are satisfied. Let $K=K(0, \rho)$ denote the closed ball in $A C \times V$ with $\rho \geq M$ centered at 0 , where $M>0$ is given in (2.1.7). If the map $g$, defined in (2.1.8) is essential in $K$ then $S\left(f, l_{i}, \psi_{i}\right) \neq \emptyset$.

Proof. Assume that $g: K \rightarrow A C \times \mathbb{R}^{n r}$ is essential. For any $(x, u) \in K$ and $v \in \psi_{r}(x, u)$ we define a function $y(x, u, v):[0,1] \rightarrow \mathbb{R}^{n}$ as follows

$$
y(x, u, v)(t)=v+\int_{0}^{t} f(\tau, x(\tau), u(\tau)) d \tau .
$$

Let $\widetilde{\varphi}: K \rightarrow A C$ given by

$$
\widetilde{\varphi}(x, u)=\left\{y(x, u, v) \mid v \in \psi_{r}(x, u)\right\} .
$$

It follows from (1.3.1) and (2.1.5) that $\widetilde{\varphi}$ is admissible. Then, in view of (2.1.1), (2.1.2), (2.1.3) and (2.1.5) again we deduce that $\widetilde{\varphi}$ is compact. Consequently, the map $\varphi: K \multimap A C \times \mathbb{R}^{n r}$ given by $\varphi(x, u)=\widetilde{\varphi}(x, u) \times \psi_{r}(x, u) \times \ldots \times \psi_{1}(x, u)$ is compact and admissible and hence $\varphi \in A^{C}\left(K, A C \times \mathbb{R}^{n r}\right)$. Let us consider the multivalued map

$$
\eta: K \multimap A C \times \mathbb{R}^{n r}
$$

defined as follows

$$
\eta(x, u)=g(x, u)-\varphi(x, u) .
$$

It follows from (1.3.2) that $\eta \in A_{\partial K}\left(K, A C \times \mathbb{R}^{n r}\right)$. We claim that $\eta$ is essential (with respect to $A^{0}\left(K, A C \times \mathbb{R}^{n r}\right)$ ). For this we define the following homotopy

$$
H: K \times[0,1] \multimap A C \times \mathbb{R}^{n r}
$$

given by

$$
H((x, u), \mu)=g(x, u)-\mu \varphi(x, u) .
$$

Then (2.1.7) implies that $H$ satisfies the assumptions of the homotopy property (compare (1.6.5)) and since $g$ is essential we get that $\eta$ is essential too. Thus by using the existence property for the map $\eta$ we get a point $(\widetilde{x}, \widetilde{u})$ in the interior of $K$ such that $0 \in \eta(\widetilde{x}, \widetilde{u})$. Hence $g(\widetilde{x}, \widetilde{u}) \in \varphi(\widetilde{x}, \widetilde{u})$ and so $l_{i}(\widetilde{x}, \widetilde{u}) \in \psi_{i}(\widetilde{x}, \widetilde{u})$, $i=1, \ldots, r$, and

$$
\widetilde{x}(t)=y(\widetilde{x}, \widetilde{u}, v)(t)=v+\int_{0}^{t} f(\tau, \widetilde{x}(\tau), \widetilde{u}(\tau)) d \tau
$$

for some $v \in \psi_{r}(\widetilde{x}, \widetilde{u})$ and for all $t \in[0,1]$. It means that $\widetilde{x}(0)=v \in \psi_{r}(\widetilde{x}, \widetilde{u})$ and

$$
\left\{\begin{array}{l}
\dot{\tilde{x}}(t)=f(t, \widetilde{x}(t), \widetilde{u}(t)), \\
l_{i}(\widetilde{x}, \widetilde{u}) \in \psi_{i}(\widetilde{x}, \widetilde{u})
\end{array} \quad \text { for } i=1, \ldots, r .\right.
$$

Therefore $(\widetilde{x}, \widetilde{u}) \in S\left(f, l_{i}, \psi_{i}\right)$ and the proof is complete.

REMARK 2.1.10. The crucial assumptions in Theorem 2.1.9 are represented by the a priori bounds (2.1.7) on the solution pairs $(x, u)$ of $(2.1 .6)_{\mu}$ and by the 
essentiality of the map $g$ defined in (2.1.8). In order to verify such conditions a suitable choice of the finite dimensional control space $V$ is fundamental. Indeed, as it is shown by the following examples if $\operatorname{dim} V=n r$ then it is possible, taking into account the assumptions on the dynamics $f$, to choose a control space $V$ which allows us to verify all the conditions of Theorem 2.1.9.

Finally, observe that under the assumptions on $f$ it is enough to verify the required a priori bounds in the $C$-space instead of the $A C$-space.

EXAmple 2.1.11. To illustrate how Theorem 2.1.9 can be used we propose the following simple but significant example. Consider the scalar boundary value control problem

$$
\left\{\begin{array}{l}
\dot{x}(t)=f(t, x(t), u(t)), \\
l_{1}(x, u) \in \psi_{1}(x, u), \\
l_{2}(x, u) \in \psi_{2}(x, u), \\
x(0) \in \psi_{2}(x, u),
\end{array}\right.
$$

where $f:[0,1] \times \mathbb{R} \times \mathbb{R} \rightarrow \mathbb{R}$ and $\psi_{i}: A C \times V \rightarrow \mathbb{R}, i=1,2$, satisfy the conditions stated before with $f$ continuous, and

$$
\begin{aligned}
& l_{1}(x, u)=x(0)+\int_{0}^{1 / 2} f(t, x(t), u(t)) d t \\
& l_{2}(x, u)=x(0)+\int_{0}^{1} f(t, x(t), u(t)) d t .
\end{aligned}
$$

As the control space $V$ we choose the following one:

$$
V=\left\{u \in L_{1} \mid u(t)=a \chi_{[0,1 / 2)}(t)+b \chi_{[1 / 2,1]}(t), a, b \in \mathbb{R}\right\}
$$

of dimension 2. Here $\chi_{A}$ denote the characteristic function of the set $A$. Observe that in this case $r=2$.

Therefore by using controls in $V$ and starting from a given set $\psi_{2}(x, u)$ we want to get at the prescribed time $t=1 / 2$ the assigned target $\psi_{1}(x, u)$ and to come back to $\psi_{2}(x, u)$ at time $t=1$. For this we posit the following conditions.

$\left(\mathrm{H}_{1}\right)$ There exists a positive constant $C$ such that $\left|\psi_{i}(x, u)\right| \leq C$ for $i=1,2$ and any $(x, u) \in A C \times V$. Here $\left|\psi_{i}(x, u)\right|=\sup _{y \in \psi_{i}(x, u)}|y|$.

$\left(\mathrm{H}_{2}\right)$ There exist positive constants $\lambda, \Lambda$ such that

$$
\lambda\left|u_{1}-u_{2}\right| \leq\left|f\left(t, x, u_{1}\right)-f\left(t, x, u_{2}\right)\right|
$$

for any $(t, x) \in[0,1] \times \mathbb{R}$ and any $u_{1}, u_{2} \in \mathbb{R}$, and

$$
\left|f\left(t, x_{1}, u\right)-f\left(t, x_{2}, u\right)\right| \leq \Lambda\left|x_{1}-x_{2}\right|
$$

for any $(t, u) \in[0,1] \times \mathbb{R}$ and any $x_{1}, x_{2} \in \mathbb{R}$. Moreover, $f(t, 0,0)=0$ for any $t \in[0,1]$. 
$\left(\mathrm{H}_{3}\right)$ For any $\rho \in \mathbb{R}$ there exists $\alpha>0$ such that

$$
\liminf _{u \rightarrow+\infty} \frac{f(t, x, u)}{u} \geq \alpha
$$

uniformly with respect to $t \in[0,1]$ and $x \geq \rho$. Futhermore,

$$
\liminf _{u \rightarrow-\infty} \frac{f(t, x, u)}{u} \geq \alpha
$$

uniformly with respect to $t \in[0,1]$ and $x \leq \rho$.

Under conditions $\left(\mathrm{H}_{1}\right)-\left(\mathrm{H}_{3}\right)$ we can prove both the existence of a ball $K \subset$ $A C \times V$ containing all the solution pairs $(x, u)$ of the problem

$$
\left\{\begin{array}{l}
\dot{x}(t)=\mu f(t, x(t), u(t)), \\
l_{1}(x, u) \in \mu \psi_{1}(x, u), \\
l_{2}(x, u) \in \mu \psi_{2}(x, u), \\
x(0) \in \mu \psi_{2}(x, u),
\end{array}\right.
$$

whenever $\mu \in[0,1]$ and that the map $g: K \rightarrow A C \times \mathbb{R}^{2}$ given by

$$
g(x, u)=\left(x, l_{2}(x, u), l_{1}(x, u)\right)
$$

is an homeomorphism onto $g(K)$ and thus it is essential since $0 \in g(K)$. Let us prove first the existence of a priori bounds for $(2.1 .12)_{\mu}$. For this observe that if the controls $u$ are bounded then from $\left(\mathrm{H}_{1}\right)-\left(\mathrm{H}_{2}\right)$ the corresponding states $x$ are also bounded. Hence we show that the controls are bounded, we argue by contradiction thus we assume the existence of sequences $\left\{\mu_{n}\right\} \subset[0,1],\left\{a_{n}\right\} \subset \mathbb{R}$ (or $\left\{b_{n}\right\} \subset \mathbb{R}$ ) such that $\lim _{n \rightarrow \infty}\left|a_{n}\right|=\infty$ (or $\lim _{n \rightarrow \infty}\left|b_{n}\right|=\infty$ ). Assume that $a_{n} \rightarrow+\infty$ as $n \rightarrow \infty$ then by $\left(\mathrm{H}_{3}\right)$ we obtain

$$
\begin{aligned}
\liminf _{n \rightarrow \infty} l_{1}\left(x_{n}, u_{n}\right) & =\liminf _{n \rightarrow \infty}\left[x_{n}(0)+\int_{0}^{1 / 2} f\left(t, x_{n}(t), a_{n}\right) d t\right] \\
& \geq x(0)+\int_{0}^{1 / 2} \liminf _{n \rightarrow \infty} f\left(t, x_{n}(t), a_{n}\right) d t=+\infty .
\end{aligned}
$$

Thus for $n$ sufficiently large $l_{1}\left(x_{n}, u_{n}\right) \notin \mu_{n} \psi_{1}\left(x_{n}, u_{n}\right)$ by $\left(\mathrm{H}_{1}\right)$. If $a_{n} \rightarrow-\infty$ again by condition $\left(\mathrm{H}_{3}\right)$ we obtain

$$
\limsup _{n \rightarrow \infty} l_{1}\left(x_{n}, u_{n}\right) \leq x(0)+\int_{0}^{1 / 2} \limsup _{n \rightarrow \infty} f\left(t, x_{n}(t), a_{n}\right) d t=-\infty .
$$

Similarly we proceed in the case when $\left\{a_{n}\right\}$ is bounded and $\left\{b_{n}\right\}$ is unbounded by using $l_{2}(x, u)$. Therefore in any case $\left(x_{n}, u_{n}\right)$, for $n$ sufficiently large, cannot be solution of $(2.1 .12)_{\mu_{n}}$. Consider now $g(x, u)=\left(x, l_{2}(x, u), l_{1}(x, u)\right)$. Clearly, 
if $x \not \equiv \widehat{x}$ we have $g(x, u) \neq g(\widehat{x}, u)$ for any $u \in V$. Assume now $u \not \equiv \widehat{u}$, this implies $a \neq \widehat{a}$ or $b \neq \widehat{b}$. Let $\widehat{a} \neq a$ and consider

$$
l_{1}(x, u)-l_{1}(x, \widehat{u})=\int_{0}^{1 / 2}[f(t, x(t), a)-f(t, x(t), \widehat{a})] d t \neq 0,
$$

where the inequality follows from $\left(\mathrm{H}_{2}\right)$. The same argument is applied to $l_{2}(x, u)$ if $a=\widehat{a}$ and $b \neq \widehat{b}$. In conclusion $g$ is one-to-one, continuous with its inverse and so it is essential since $0 \in g(K)$ with $K \subset A C \times V$ ball of sufficiently large radius.

Observe that, under assumptions $\left(\mathrm{H}_{1}\right)-\left(\mathrm{H}_{3}\right)$, the same arguments as before can be easily adapted to the more general situation

$$
\left\{\begin{array}{c}
\dot{x}(t)=f(t, x(t), u(t)), \\
l_{1}(x, u) \in \psi_{1}(x, u), \\
l_{2}(x, u) \in \psi_{2}(x, u), \\
\vdots \\
\vdots \\
l_{r-1}(x, u) \in \psi_{r-1}(x, u), \\
l_{r}(x, u) \in \psi_{r}(x, u), \\
x(0) \in \psi_{r}(x, u),
\end{array}\right.
$$

where $l_{i}(x, u)=x(0)+\int_{0}^{t_{i}} f(t, x(t), u(t)) d t, 0<t_{1}<\ldots<t_{r}=1$, and the control space of dimension $r$ is defined by

$$
V=\left\{u \in L_{1} \mid u=a_{1} \chi_{\left[0, t_{1}\right)}(t)+a_{2} \chi_{\left[t_{1}, t_{2}\right)}(t)+\ldots+a_{r} \chi_{\left[t_{r-1}, 1\right]}(t)\right\},
$$

where $a_{i} \in \mathbb{R}, i=1, \ldots, r$.

Finally, in a similar way we can solve (2.1.12) when $x(t)$ and $u(t)$ are vectors of $\mathbb{R}^{n}, n>1$, by replacing assumptions $\left(\mathrm{H}_{2}\right)$ and $\left(\mathrm{H}_{3}\right)$ with the following ones.

$\left(\mathrm{H}_{2}\right)^{\prime}$ There exist positive constants $\lambda, \Lambda$ such that

$$
\lambda\left|u_{1}-u_{2}\right|^{2} \leq\left(u_{1}-u_{2}, f\left(t, x, u_{1}\right)-f\left(t, x, u_{2}\right)\right)
$$

for any $(t, x) \in[0,1] \times \mathbb{R}^{n}$ and any $u_{1}, u_{2} \in \mathbb{R}^{n}$, and

$$
\left|f\left(t, x_{1}, u\right)-f\left(t, x_{2}, u\right) \leq \Lambda\right| x_{1}-x_{2} \mid
$$

for any $(t, u) \in[0,1] \times \mathbb{R}^{n}$ and any $x_{1}, x_{2} \in \mathbb{R}^{n}$. Moreover, $f(t, 0,0)=0$ for any $t \in[0,1]$. Here $(\cdot, \cdot)$ denotes the usual scalar product in $\mathbb{R}^{n}$.

$\left(\mathrm{H}_{3}\right)^{\prime}$ There exists a positive constant $\alpha$ such that for any $i \in\{1, \ldots, n\}$ there exists $j \in\{1, \ldots, n\}$ for which

$$
\liminf _{u_{i} \rightarrow \pm \infty} \frac{f_{j}\left(t, x, u_{1}, \ldots, u_{i}, \ldots, u_{n}\right)}{u_{i}} \geq \alpha
$$

uniformly with respect to the other variables. 
and choosing as $2 n$-dimensional control space $V=\left\{u \in L_{1} \mid u(t)=a \chi_{[0,1 / 2)}(t)+\right.$ $\left.b \chi_{[1 / 2,1]}(t) ; a, b \in \mathbb{R}^{n}\right\}$. The details are left to the reader.

2.2. Perturbed linear control systems. Now, we will consider boundary value problems for perturbed linear control system. Here we consider a different homotopy $(2.2 .2)_{\mu}$ which leads to a different definition of $g$, i.e. in this case $g(x, u)=\left(\dot{x}, l_{r}(x, u), \ldots, l_{1}(x, u)\right)$. As we will show in the sequel this permits to avoid the extra condition on the initial state $x(0) \in \psi_{r}(x, u)$. Indeed, this condition represents a further boundary condition to be added to the $r$ assigned boundary conditions $\left(l_{r}(x, u), \ldots, l_{1}(x, u)\right)$ of the problem. As a consequence, by means of the homotopy $(2.2 .2)_{\mu}$, we can treat control problems for which the initial state $x(0)$ does not necessarily satisfy preassigned constraints. An example of this situation is represented by periodic control problems, which will be treated in the next Example 2.2.10.

Assume that $f_{1}:[0,1] \times \mathbb{R}^{n} \times \mathbb{R}^{m} \rightarrow \mathbb{R}^{n}$ satisfies (2.1.1), (2.1.2) and the following condition.

(2.2.1) There exists a (Lebesque) integrable function $\alpha:[0,1] \rightarrow[0,+\infty)$ such that $\left|f_{1}(t, x, u)\right| \leq \alpha(t)$ for all $(x, u) \in \mathbb{R}^{n} \times \mathbb{R}^{m}$ and a.a. $t \in[0,1]$.

Let $A(\cdot)($ resp. $B(\cdot))$ be a time dependent $n \times n$ (respectively, $n \times m$ ) matrix with (Lebesque) integrable coefficients with values in $\mathbb{R}$. Assume that $l_{i}$, $\psi_{i}, i=1, \ldots, r$, are mapping satisfying (2.1.4) and (2.1.5) respectively. Consider the following problem

$$
\left\{\begin{array}{l}
\dot{x}(t)=A(t) x(t)+B(t) u(t)+f_{1}(t, x(t), u(t)), \\
l_{i}(x, u) \in \psi_{i}(x, u)
\end{array} \text { for } i=1, \ldots, r,\right.
$$

and for any $\mu \in[0,1]$ consider

$(2.2 .2)_{\mu} \begin{cases}\dot{x}(t)=\mu\left[A(t) x(t)+B(t) u(t)+f_{1}(t, x(t), u(t))\right], & \\ l_{i}(x, u) \in \mu \psi_{i}(x, u) & \text { for } i=1, \ldots, r .\end{cases}$

As in Section 2.1 we need the following assumption.

(2.2.3) There exists $M>0$ such that for any $\mu \in[0,1]$ and any solution pair $(x, u)$ of $(2.2 .2)_{\mu}$ we have $\|(x, u)\|<M$.

We associate with (2.2.2) the following map

$$
\left\{\begin{array}{l}
g: A C \times V \rightarrow L_{1} \times \mathbb{R}^{n r}, \\
g(x, u)=\left(\dot{x}, l_{r}(x, u), \ldots, l_{1}(x, u)\right) .
\end{array}\right.
$$

Finally, let $K$ be a closed ball with center 0 and radius $\rho \geq M$.

We are in a position to prove the following. 
Theorem 2.2.5. Let $f_{1}, A(\cdot), B(\cdot), l_{i}, \psi_{i}, i=1, \ldots, r$, be as above. Assume further that the map $g$ defined in (2.2.4) is an essential map in some ball $K$. Then (2.2.2) has a solution.

Proof. Assume that $g: K \rightarrow L_{1} \times \mathbb{R}^{n r}$ is essential. Define a map $\varphi_{1}: K \rightarrow$ $L_{1}$ by

$$
\varphi_{1}(x, u)(t)=A(t) x(t)+B(t) u(t)+f_{1}(t, x(t), u(t))
$$

for any $(x, u) \in K$ and $t \in[0,1]$.

It is proved in ([21], p. 236) that $\varphi_{1}$ is a compact map. This allows us to define a compact admissible map $\varphi: K \multimap L_{1} \times \mathbb{R}^{n r}$ by the formula

$$
\varphi(x, u)=\varphi_{1}(x, u) \times \psi_{r}(x, u) \times \ldots \times \psi_{1}(x, u)
$$

for any $(x, u) \in K$. Let $\eta: K \multimap L_{1} \times \mathbb{R}^{n r}$ be defined as follows

$$
\eta(x, u)=g(x, u)-\varphi(x, u) .
$$

In view of the existence property it is sufficient to show that $\eta$ is essential. We show this in a similar way of the proof of Theorem 2.1.9. namely, we consider the homotopy

$$
H: K \times[0,1] \multimap L_{1} \times \mathbb{R}^{n r}, \quad H((x, u), \mu)=g(x, u)-\mu \varphi(x, u) .
$$

Then (2.2.3) guarantees that $H$ is an admissible homotopy joining $g$ with $\eta$ which satisfies (1.6.5). Thus from the homotopy property follows that $\eta$ is essential since $g$ is. This completes the proof.

EXAmPLe 2.2.6. Consider the following classical controllability problem for the perturbed linear control system (2.2.2).

$$
\left\{\begin{array}{l}
\dot{x}(t)=A(t) x(t)+B(t) u(t)+f_{1}(t, x(t), u(t)), \\
x(1)=x_{1}, \\
x(0)=0 .
\end{array}\right.
$$

Here $\psi_{1}(x, u)=\left\{x_{1}\right\}, \psi_{2}(x, u)=\{0\}$ and $l_{1}(x, u)=x(0)+\int_{0}^{1} f(t, x(t), u(t)) d t$, $l_{2}(x, u)=x(0)$. Observe that the boundary condition $x(0)=0$ represents here the extra condition $x(0) \in \psi_{2}(x, u)$ of Section 2.1, thus we will choose a control space $V$ of dimension $n$. Consider the associated linear control problem

$$
\left\{\begin{array}{l}
\dot{x}(t)=A(t) x(t)+B(t) u(t), \\
x(1)=x_{1} \\
x(0)=0 .
\end{array}\right.
$$

We assume the following conditions

$\left(\mathrm{L}_{1}\right)$ the linear control system $(2.2 .8)$ is completely controllable from zero, that is for any $x_{1} \in \mathbb{R}^{n}$ there exists a control function $u \in U_{B}=\{u \in$ 
$\left.L_{1} \mid B(\cdot) u(\cdot) \in L_{1}\right\}$ such that the corresponding solution $x=x(t)$ satisfies $x(1)=x_{1}$.

$\left(\mathrm{f}_{1}\right) \lim _{|x|+|u| \rightarrow \infty}\left|f_{1}(t, x, u)\right| /(|x|+|u|)=0$ uniformly for $t \in[0,1]$.

In [20] it was shown that (2.2.8) is still completely controllable by means of the $n$-dimensional control space $V$ defined as

$$
V=\operatorname{span}\left\{\chi_{t_{1}}^{i_{1}}, \ldots, \chi_{t_{n}}^{i_{n}}\right\}
$$

where $\chi_{t_{j}}^{i_{j}}(t)=\chi_{\left[0, t_{j}\right)}(t) e_{i_{j}}, j=1, \ldots, n$, and $\left\{e_{k}\right\}_{k=1}^{m}$ is the canonical basis of $\mathbb{R}^{m}$ with the indexes $i_{j} \in\{1, \ldots, m\}$ such that

$$
\operatorname{det}\left(X^{-1}\left(t_{1}\right) \widetilde{x}_{i_{1}}\left(t_{1}\right), \ldots, X^{-1}\left(t_{n}\right) \widetilde{x}_{i_{n}}\left(t_{n}\right)\right) \neq 0,
$$

where $\widetilde{x}_{i_{j}}(t), j=1, \ldots, n$, is the solution of the problem

$$
\left\{\begin{array}{l}
\dot{x}(t)=A(t) x(t)+B(t) e_{i_{j}}, \\
x(0)=0
\end{array}\right.
$$

and $X(t)$ is the fundamental matrix of $\dot{x}(t)=A(t) x(t)$. Indeed, it is possible to show that such a choice makes the boundary condition

$$
l_{1}(x, u)=X(1) \int_{0}^{1} X^{-1}(s) B(s) u(s) d s
$$

an isomorphism between $V$ and $\mathbb{R}^{n}$, see [20] for the details. Therefore, if we consider the homotopy

$$
\left\{\begin{array}{l}
\dot{x}(t)=\mu[A(t) x(t)+B(t) u(t)], \\
X(1) \int_{0}^{1} X^{-1}(s) B(s) u(s) d s=\mu x_{1}, \\
x(0)=0,
\end{array}\right.
$$

where $\mu \in[0,1]$, it can be show that the associated linear operator $L_{\mu}: A C \times V \rightarrow$ $L^{1} \times \mathbb{R}^{2 n}$ given by

$$
L_{\mu}(x, u)(t)=\left(\dot{x}(t)-\mu[A(t) x(t)+B(t) u(t)], l_{2}(x, u), l_{1}(x, u)\right)
$$

is an isomorphism for any $\mu \in[0,1]$. Namely, there exists $c>0$ such that

$$
\begin{aligned}
c\left(\max _{t \in[0,1]}|x(t)|+\|u\|_{L_{1}}\right) \leq \| \dot{x}(\cdot)-\mu[A(\cdot) x(\cdot) & +B(\cdot) u(\cdot)] \|_{L_{1}} \\
& +\left|l_{1}(x, u)\right|+\left|l_{2}(x, u)\right| .
\end{aligned}
$$

Observe that by $\left(f_{1}\right)$, the a priori bounds of $x$ in $C$ together with the boundedness of the controls in the finite dimensional space $V$ imply a priori bounds of $x$ in $A C$, so we have the required bounds on the solution pairs $(x, u)$ of $(2.2 .8)_{\mu}$. In particular for $\mu=0$ we obtain that $g(x, u)=\left(\dot{x}, l_{1}(x, u), l_{2}(x, u)\right)$ is an isomorphism. 
Returning to the homotopy considered in Theorem 2.2.5 we have for the considered system

$$
\left\{\begin{array}{l}
\dot{x}(t)=\mu\left[A(t) x(t)+B(t) u(t)+f_{1}(t, x(t), u(t))\right], \\
l_{1}(x, u)=\mu x_{1}, \\
l_{2}(x, u)=0 .
\end{array}\right.
$$

From (2.2.9) and $\left(f_{1}\right)$ we can now derive a priori bounds for $(2.2 .7)_{\mu}$. In fact $\left(f_{1}\right)$ ensures that for any $\varepsilon>0$ there exists $\rho=\rho(\varepsilon)>0$ such that for $(|x|+|u|)>\rho$ and a.a. $t \in[0,1]$ we have

$$
\left|f_{1}(t, x, u)\right| \leq \varepsilon(|x|+|u|)
$$

and so there exists $\gamma_{\rho} \in L_{1}$ such that

$$
\left|f_{1}(t, x, u)\right| \leq \gamma_{\rho}(t)+\varepsilon(|x|+|u|)
$$

for any $(x, u) \in \mathbb{R}^{n} \times \mathbb{R}^{m}$ and a.a. $t \in[0,1]$. In conclusion, if $(x, u)$ is any solution of $(2.2 .7)_{\mu}$ we have

$$
\begin{aligned}
c\left(\max _{t \in[0,1]}|x(t)|+\|u\|_{L_{1}}\right) & \leq \mu\left(\|f(\cdot, x(\cdot), u(\cdot))\|_{L_{1}}+\left|x_{1}\right|\right) \\
& \leq\left\|\gamma_{\rho}\right\|_{L_{1}}+\left|x_{1}\right|+\varepsilon\left(\max _{t \in[0,1]}|x(t)|+\|u\|_{L_{1}}\right)
\end{aligned}
$$

and for $0<\varepsilon<c$ and $x_{1}$ in a bounded set we get the a priori bounds for the solutions of $(2.2 .7)_{\mu}$ which together with the fact that $g$ is an isomorphism guarantee the solvability of (2.2.7) whenever $x_{1} \in \mathbb{R}^{n}$ assigned.

Note that the same arguments also show the solvability of

$$
\left\{\begin{array}{l}
\dot{x}(t)=A(t) x(t)+B(t) u(t)+f_{1}(t, x(t), u(t)), \\
l_{1}(x, u)=x_{1}, \\
l_{2}(x, u)=x_{0},
\end{array}\right.
$$

for any $x_{0}, x_{1} \in \mathbb{R}^{n}$. In fact the complete controllability of (2.2.8) from zero is equivalent to the complete controllability from any other initial point $x_{0} \in \mathbb{R}^{n}$.

ExAmPle 2.2.10. As a further perturbed linear control problem we consider the following periodic control system

$$
\left\{\begin{array}{l}
\dot{x}(t)=A(t) x(t)+B(t) u(t)+f_{1}(t, x(t), u(t)):=f(t, x(t), u(t)), \\
l_{1}^{i}(x, u):=\left(\int_{0}^{1} u_{i}(t) d t\right) \in\left[-\varphi_{i}(x, u), \varphi_{i}(x, u)\right], \quad i=1, \ldots, m \\
l_{2}(x, u):=\int_{0}^{1} f(t, x(t), u(t)) d t=0
\end{array}\right.
$$

where $\varphi_{i}: A C \times V \rightarrow \mathbb{R}_{+}, i=1, \ldots, m$, are continuous function for which there exists a constant $C>0$ such that $\varphi_{i}(x, u) \leq C$ for any $i=1, \ldots, m$ and any pair $(x, u) \in A C \times V$. Thus $\psi_{1}(x, u)=\left[-\varphi_{1}(x, u), \varphi_{1}(x, u)\right] \times \ldots \times$ 
$\left[-\varphi_{m}(x, u), \varphi_{m}(x, u)\right]$ and $\psi_{2}(x, u)=\{0\}$ for any $(x, u) \in A C \times V$. We assume here $V=\mathbb{R}^{m}$. In what follows $(\cdot, \cdot)$ denotes the usual scalar product in $\mathbb{R}^{n}$. We assume the following conditions on the dynamics $f$.

$\left(\mathrm{A}_{1}\right)$ There exist positive constants $\alpha, \Lambda$ such that

$$
\left|f\left(t, x_{1}, u\right)-f\left(t, x_{2}, u\right)\right| \leq \Lambda\left|x_{1}-x_{2}\right|
$$

and

$$
\left(x_{1}-x_{2}, f_{1}\left(t, x_{1}, u\right)-f_{1}\left(t, x_{2}, u\right)\right) \leq \alpha\left|x_{1}-x_{2}\right|^{2}
$$

for a.a. $t \in[0,1]$, any $u \in \mathbb{R}^{m}$ and $x_{1}, x_{2} \in \mathbb{R}^{n}$.

$\left(\mathrm{A}_{2}\right) \lim _{|x|+|u| \rightarrow \infty}\left|f_{1}(t, x, u)\right| /(|x|+|u|)=0$ uniformly in $t \in[0,1]$.

$\left(\mathrm{A}_{3}\right) \int_{0}^{1} \sup _{|x|=1}(x, A(t) x) d t<-\alpha$, with $\alpha>0$ the same constant of $\left(\mathrm{A}_{1}\right)$.

We associate to (2.2.11) the family of problems with $\mu \in[0,1]$

$$
\left\{\begin{array}{l}
\dot{x}(t)=\mu f(t, x(t), u(t)) \\
l_{1}^{i}(x, u) \in\left[-\mu \varphi_{i}(x, u), \mu \varphi_{i}(x, u)\right] \text { for } i=1, \ldots, m \\
l_{2}(x, u)=0
\end{array}\right.
$$

Clearly, the boundary conditions $l_{1}^{i}(x, u) \in\left[-\mu \varphi_{i}(x, u), \mu \varphi_{i}(x, u)\right]$, for $i=$ $1, \ldots, m$, guarantee the boundedness of the controls in $V=\mathbb{R}^{m}$. If we assume the existence of sequences $\left\{\mu_{n}\right\} \subset[0,1],\left(x_{n}, u_{n}\right) \in A C \times V$ such that $\left(x_{n}, u_{n}\right)$ solves $(2.2 .11)_{\mu_{n}}$ and $\max _{t \in[0,1]}\left|x_{n}(t)\right| \rightarrow \infty$, (as already noted the boundedness of $\left\{x_{n}\right\}$ in $C$ together with that of the controls in the finite dimensional space $V$ implies its boundedness in $A C$ ), then by using standard arguments it is possible to show that $\min _{t \in[0,1]}\left|x_{n}(t)\right| \rightarrow \infty$ and so by $\left(\mathrm{A}_{2}\right)-\left(\mathrm{A}_{3}\right)$ we get a contradiction. We report here some details for the reader convenience. Consider

$$
\dot{x}_{n}(t)=\mu_{n} f\left(t, x_{n}(t), u_{n}(t)\right), \quad t \in[0,1]
$$

with the condition

$$
\int_{0}^{1} f\left(t, x_{n}(t), u_{n}(t)\right) d t=0
$$

Using $\left(A_{2}\right)$ we get

$$
\begin{aligned}
\frac{d}{d t} \frac{\left|x_{n}(t)\right|^{2}}{2} & =\mu_{n}\left(x_{n}(t), f\left(t, x_{n}(t), u_{n}(t)\right)\right) \\
& =\mu_{n}\left(x_{n}(t), A(t) x_{n}(t)+B(t) u_{n}(t)+f_{1}\left(t, x_{n}(t), u_{n}(t)\right)\right) \\
& \leq a(t)\left|x_{n}(t)\right|^{2}+b(t)\left|x_{n}(t)\right|
\end{aligned}
$$


for some $a, b \in L_{1}$. Dividing by $1+\left|x_{n}(t)\right|^{2}$, integrating on the interval $[0,1]$ and using the 1-periodicity of $x_{n}$ we get

$$
\log \max _{t \in[0,1]}\left(1+\left|x_{n}(t)\right|^{2}\right) \leq \log \min _{t \in[0,1]}\left(1+\left|x_{n}(t)\right|^{2}\right)+A,
$$

where

$$
A \geq \int_{0}^{1}\left[a(t)+\frac{b(t)\left|x_{n}(t)\right|}{1+\left|x_{n}(t)\right|^{2}}\right] d t .
$$

Thus $\max _{t \in[0,1]}\left|x_{n}(t)\right| \rightarrow \infty$ implies $\min _{t \in[0,1]}\left|x_{n}(t)\right| \rightarrow \infty$ as $n \rightarrow \infty$. Therefore for $n$ sufficiently large if $\mu_{n}>0$ integrating on $[0,1]$ the first equality in $(2.2 .13)$ we obtain

$$
\int_{0}^{1} \frac{\left(x_{n}(t), f\left(t, x_{n}(t), u_{n}(t)\right)\right)}{\left|x_{n}(t)\right|^{2}} d t=0 .
$$

On the other hand if $\mu_{n}=0$ then $x(t)=$ const. for any $t \in[0,1]$ and the previous equation is a direct consequence of (2.2.12). Now by $\left(\mathrm{A}_{2}\right)-\left(\mathrm{A}_{3}\right)$, the boundedness of $\left\{u_{n}\right\} \subset V$ and the fact that $\min _{t \in[0,1]}\left|x_{n}(t)\right| \rightarrow \infty$ we get

$$
\begin{aligned}
0 & =\limsup _{n \rightarrow \infty} \int_{0}^{1} \frac{\left(x_{n}(t), f\left(t, x_{n}(t), u_{n}(t)\right)\right)}{\left|x_{n}(t)\right|^{2}} d t \\
& \leq \int_{0}^{1} \limsup _{n \rightarrow \infty} \frac{\left(x_{n}(t), f\left(t, x_{n}(t), u_{n}(t)\right)\right)}{\left|x_{n}(t)\right|^{2}} d t \leq \int_{0}^{1} \sup _{|x|=1}(x, A(t) x) d t \leq-\alpha<0,
\end{aligned}
$$

which is a contradiction. This proves the boundedness of the states $x$.

Finally, the map $g(x, u)=\left(\dot{x}, l_{2}(x, u), l_{1}(x, u)\right)$ is one-to-one. In fact if $u \neq \widehat{u}$, $u, \widehat{u} \in \mathbb{R}^{m}$, then $l_{1}(x, u) \neq l_{1}(x, \widehat{u})$ for any $x \in A C$ where $l_{1}=\left(l_{1}^{i}\right)_{i=1}^{m}$. Let $x, \widehat{x} \in A C$ with $x \not \equiv \widehat{x}$, we have two possibilities: $\dot{x}(t) \neq \dot{\hat{x}}(t)$ on a positive measure set $E \subset[0,1]$ or $\dot{x}(t)=\dot{\widehat{x}}(t)$ for a.a. $t \in[0,1]$. We have to consider only this last possibility, in this case $x(t)-\widehat{x}(t)=$ const. $=q \neq 0, t \in[0,1]$. For $u \in V$ we calculate

$$
l_{2}(x, u)-l_{2}(\widehat{x}, u)=\int_{0}^{1} A(t) q+\left[f_{1}(t, \widehat{x}(t)+q, u(t))-f_{1}(t, \widehat{x}(t), u(t))\right] d t
$$

and so by $\left(A_{1}\right)$ and $\left(A_{3}\right)$ we obtain

$$
\left(q, l_{2}(x, u)-l_{2}(\widehat{x}, u)\right) /|q|^{2}<0,
$$

thus $g$ is one-to-one. On the hand $g: K \subset A C \times V \rightarrow L_{1} \times \mathbb{R}^{2 n}$ is continuous as well as its inverse, as it is easily seen, and $0 \in g(K)$. Therefore, $g$ is essential. Observe that we can substitute in $\left(A_{1}\right)$ and $\left(A_{3}\right)$ the following conditions

$$
\left(x_{1}-x_{2}, f_{1}\left(t, x_{1}, u\right)-f_{1}\left(t, x_{2}, u\right)\right) \geq \alpha\left|x_{1}-x_{2}\right|^{2}
$$

and

$$
\int_{0}^{1} \inf _{|x|=1}(x, A(t) x) d t>-\alpha,
$$

respectively, obtaining the same conclusion. 
2.3. $k$ th order boundary value control problems. In this part we would like to point out that our method presented in the previous sections works without any substantial change in the case of $k$ th order control problems. For simplicity we will restrict our considerations to the boundary conditions of Section 2.1. Assume that $f:[0,1] \times \mathbb{R}^{n k} \times \mathbb{R}^{m} \rightarrow \mathbb{R}^{n}$ satisfies (2.1.1)-(2.1.3), here the second argument is considered as a vector in $\mathbb{R}^{n k}, k>1$. Assume further that

$$
l_{i}: C^{k-1} \times V \rightarrow \mathbb{R}^{n}, \quad i=1, \ldots, r, \text { is a continuous map, }
$$

and

$$
\begin{aligned}
\psi_{i}: C^{k-1} \times V \multimap \mathbb{R}^{n}, \quad i=1, \ldots, r, \text { is an admissible } \\
\text { completely continuous map. }
\end{aligned}
$$

We want to study the following problem

$$
\begin{cases}x^{(k)}(t)=f\left(t, x(t), \dot{x}(t), \ldots, x^{k-1}(t), u(t)\right) & \text { for } t \in[0,1], \\ l_{i}(x, u) \in \psi_{i}(x, u) & \text { for } i=1, \ldots, r \\ x^{(k-1)}(0) \in \psi_{r}(x, u) . & \end{cases}
$$

Let $S^{k}\left(f, l_{i}, \psi_{i}\right)$ the set of all the solutions of $(2.3 .3)$ and $S_{\mu}^{k}\left(f, l_{i}, \psi_{i}\right)$ the set of all the solutions $(x, u)$ of the following problem

$(2.3 .3)_{\mu} \begin{cases}x^{(k)}(t)=\mu f\left(t, x(t), \dot{x}(t), \ldots, x^{k-1}(t), u(t)\right) & \text { for } t \in[0,1], \\ l_{i}(x, u) \in \mu \psi_{i}(x, u) & \text { for } i=1, \ldots, r \\ x^{(k-1)}(0) \in \mu \psi_{r}(x, u) & \end{cases}$

for $\mu \in[0,1]$. We assume that

(2.3.4) there exists $M>0$ such that for any $\mu \in[0,1]$ and any $(x, u) \in$ $S_{\mu}^{k}\left(f, l_{i}, \psi_{i}\right)$ we have $\|(x, u)\|<M$.

Let associate to (2.3.3) the map $g: C^{k-1} \times V \rightarrow C \times \mathbb{R}^{n r}$ defined by

$$
g(x, u)=\left(x^{(k-1)}, l_{r}(x, u), \ldots, l_{1}(x, u)\right) .
$$

REMARK 2.3.6. If $C_{a}^{k-1}$ is the subspace of $C^{k-1}$ of all the functions with the $(k-1)$-derivative absolutely continuous and $l_{i}, \psi_{i}$ are defined on $C_{a}^{k-1} \times V$, then $g$ can be considered as a map defined on $C_{a}^{k-1} \times V$ with values in $A C \times \mathbb{R}^{n r}$.

We can prove the following theorem

THEOREM 2.3.7. Under all the above assumptions if $g$ is essential on some ball $K=K(0, \rho), \rho \geq M$, then $S^{k}\left(f, l_{i}, \psi_{i}\right) \neq \emptyset$.

The proof of Theorem 2.3.7 is quite analogous to the proof of Theorem 2.1.9, and thus we will omit it. 
EXAMPLE 2.3.8. For simplicity, we consider the following 2-order differential equation

$$
\ddot{x}(t)=h(t, x(t), \dot{x}(t))+u(t):=f(t, x(t), \dot{x}(t), u(t))
$$

where $h:[0,1] \times \mathbb{R}^{2} \rightarrow \mathbb{R}$ satisfies Carathéodory type conditions and the control function $u:[0,1] \rightarrow \mathbb{R}$ belongs to $L_{1}$. We assume that $f$ satisfies $\left(\mathrm{H}_{2}\right)-\left(\mathrm{H}_{3}\right)$ of Example 2.1.11 with $x$ replaced by the vector $X=\left(x_{1}, x_{2}\right)=(x, \dot{x})$ and the condition $x \geq \rho(x \leq \rho)$ in $\left(\mathrm{H}_{3}\right)$ by $x_{i} \geq \rho\left(x_{i} \leq \rho\right), i=1,2$.

For convenience we rewrite (2.3.9) in the form

$$
\dot{X}(t)=H(t, X(t))+U(t)),
$$

where $H(t, X)=\left(x_{2}, h\left(t, x_{1}, x_{2}\right)\right)$ and $U=(0, u)$.

We consider (2.3.10) under the boundary conditions

$$
\begin{aligned}
& L_{1}(X, U)=\left(\begin{array}{l}
x_{1}(0) \\
x_{2}(0)
\end{array}\right) \in I_{0}, \\
& L_{2}(X, U) \\
& \quad=\left(\begin{array}{c}
x_{1}(0)+x_{2}(0)+\int_{0}^{1}(1-s)\left[h\left(s, x_{1}(s), x_{2}(s)\right)+u(s)\right] d s \\
x_{2}(0)+\int_{0}^{1}\left[h\left(s, x_{1}(s), x_{2}(s)\right)+u(s)\right] d s
\end{array}\right) \in I_{1},
\end{aligned}
$$

where $I_{0}, I_{1}$ are assigned intervals of $\mathbb{R}^{2}$ with $I_{0} \subseteq I_{1}$. As a control space we consider the following 2-dimensional vector space

$$
V=\left\{u \in L_{1} \mid u(t)=a \chi_{[0,1 / 2]}(t)+b \chi_{(1 / 2,1]}(t) ; a, b \in \mathbb{R}\right\} .
$$

Now we can proceed as in Example 2.1.11 to show the boundedness of the controls. The only point is to show that the two components of the vector $X(t)$ are bounded by below on $[0,1 / 2]$ if $a_{n} \rightarrow \infty$ or upper bounded if $a_{n} \rightarrow-\infty$ in order to apply condition $\left(\mathrm{H}_{3}\right)$ to the interval $[0,1 / 2]$. The same holds for the interval $[1 / 2,1]$ if $\left\{a_{n}\right\}$ is bounded and $\left\{b_{n}\right\}$ is unbounded. Finally, we associate to (2.3.10)-(2.3.11) the map $G: A C \times V \rightarrow A C \times \mathbb{R}^{4}$ given by

$$
G(X, U)=\left(X, L_{2}(X, U), L_{1}(X, U)\right) .
$$

It is easy to show by a direct calculation that $G$ is essential on some ball $K$ of sufficiently large radius. We leave the details to the reader.

\section{REFERENCES}

[1] W. Allegretto And P. Nistri, Periodic solutions and optimization problems for a class of semilinear parabolic control systems, Topol. Methods Nonlinear Anal. 5 (1995), $345-356$.

[2] Control and optimization of nonlocal steady-state problems, Lecture Notes in Nonlinear Analysis 2 (1998), N. Copernicus University, Toruń, 9-18. 
[3] G. Anichini, Boundary value problems for multivalued equations and controllability, J. Math. Anal. Appl. 105 (1985), 372-382.

[4] G. Anichini And P. ZeCCA, Multivalued differential equations in Banach spaces. An application to control theory, J. Optim. Theory Appl. 21 (1977), 477-486.

[5] J. P. Aubin, Viability Theory, Birkhäuser, 1991.

[6] J. P. Aubin and A. Cellina, Differential inclusions, Grundlehren Math. Wiss., vol. 264, Springer Verlag, Berlin, 1984.

[7] N. Carmichael, A. J. Pritchard and N. D. Quinn, Control and state-estimate of nonlinear systems, Res. Notes Math. 68 (1982), 30-51.

[8] State and parameter estimation problems for nonlinear systems, Appl. Maths. Optim. 9 (1982), 133-161.

[9] E. N. Chukwu And J. M. Gronski, Approximate and complete controllability of nonlinear systems to a convex target set, J. Math. Anal. Appl. 61 (1977), 97-112.

[10] C. DACKA, On the controllability of a class of nonlinear systems, IEEE Trans. Automat. Control AC-25 (1980), 263-266.

[11] J. P. DAuer, A controllability technique for nonlinear systems, J. Math. Anal. Appl. 37 (1972), 442-451.

[12] _ A note on bounded perturbations of controllable systems, J. Math. Anal. Appl. 42 (1973), 221-225.

[13] - Approximate controllability of nonlinear systems with restrained controls, J. Math. Anal. Appl. 46 (1974), 126-131.

[14] Nonlinear pertubations of quasilinear control systems, J. Math. Anal. Appl. 54 (1976), 717-725.

[15] Controllability of perturbed nonlinear systems, Rend. Accad. Naz. Lincei LXIII (1977), 345-350.

[16] E. J. Davison And D. BALl, On the global controllability of perturbed controllable systems, IEEE Trans. Automat. Control AC-17 (1972), 825-826.

[17] E. J. Davison and E. G. Kunze, Some sufficient conditions for the global and local controllability of nonlinear time-varying systems, SIAM J. Control 8 (1970), 489-494.

[18] J. A. M. Felippe De Souza And A. J. Pritchard, A note on overall observability: the joint problem of state estimation and parameter identification, J. Comput. Appl. Math. 12 (1993), 19-31.

[19] A. F. Filippov, On certain questions in the theory of optimal control, SIAM J. Control Optim. 1 (1962), 76-84.

[20] M. Furi, P. Nistri, M. P. Pera and P. L. Zezza, Linear controllability by piecewise constant controls with assigned switching times, J. Optim. Theory Appl. 45 (1985), 219-229.

[21] Topological methods for the global controllability of nonlinear systems, J. Optim. Theory Appl. 45 (1985), 231-256.

[22] L. Górniewicz, Homological methods in fixed point theory of multivalued mappings, Diss. Math. 129 (1976), 1-71.

[23] L. Górniewicz And P. Nistri, An invariance problem for control systems with deterministic uncertainy, Topology in Nonlinear Analysis, vol. 35, Banach Center Publications, 1996, pp. 193-205.

[24] L. Górniewicz, P. Nistri and P. ZecCA, Control problems on closed subset on $\mathbb{R}^{n}$ via feedback controls, Topol. Methods Nonlinear Anal. 82 (1993), 163-178.

[25] L. Górniewicz and M. SlosarskiĬ, Topological essentiality and differential inclusions, Bull. Austral. Math. Soc. 45 (1992), 177-193.

[26] M. I. Kamenskil̆, P. Nistri, V. V. ObukhovskiĬ And P. Zecca, Optimal feedback control for a semilinear evolution equation, J. Optim. Theory Appl. 82 (1994), 503-517. 
[27] J. Klamka, On the global controllability of perturbed systems, IEEE Trans. Automat. Control AC-20 (1975), 170-172.

[28] W. Kryszewsini and S. Plaskacz, Topological methods for the local controllability of nonlinear systems, SIAM J. Control Optim. 32 (1994), 213-223.

[29] D. L. Lukes, Global controllability of nonlinear systems, SIAM J. Control Optim. 10 (1972), 112-126.

[30] K. Magnusson, A. J. Pritchard and N. D. Quinn, The application of fixed point theorems to global nonlinear controllability problems, Banach Center Publications, vol. 14, 1985, pp. 319-344.

[31] K. Mirza And B. F. Womack, On the controllability of a class of nonlinear systems, IEEE Trans. Automat. Control AC-17 (1972), 532-535.

[32] P. Nistri, On a general notion of controllability for nonlinear systems, Boll. Un. Mat. Ital. 5 (1986), 383-403.

[33] Optimal control problems via a direct method, Ann. Mat. Pura Appl. 69 (1991), 295-314.

[34] P. Nistri, V. V. Obukhovski sions involving noncompact operators, Trans. Amer. Math. Soc. 342 (1994), 543-562.

[35] _ Viability for feedback control systems in Banach spaces via Carathéodory closedloop controls, Differential Equations Dynam. Systems 4 (1996), 365-378.

[36] N. S. Papageorgiou, Viable and periodic solutions for differential inclusions in Banach spaces, Kobe J. Math. 5 (1988), 29-42.

[37] M. P. PERA, A topological method for solving nonlinear equations in Banach spaces and some related global results on the solution sets, Rend. Sem. Mat. Univ. Politec. Torino 41 (1983), 73-87.

[38] A. J. Pritchard, The application of fixed point theorems to nonlinear system theory, Proc. 3th. IMA Conference (1980), Sheffield, 775-792.

[39] I. Tarnove, A controllability problem for nonlinear systems, Mathematical Theory of Control (A. V. Balakrishnan and L. W. Neustadt, eds.), Academic Press Ed., New York, 1967, pp. 170-179.

[40] Y. Yамамото, Controllability of nonlinear systems, J. Optim. Theory Appl. 22 (1977), 41-49.

[41] K. C. WeI, A class of controllable nonlinear systems, IEEE Trans. Automat. Control AC-21 (1976), 787-789.

LeCh GóRNIEWICZ

Faculty of Mathematics and Informatics

Nicholas Copernicus University

Chopina 12/18, 87-100 Toruń, POLAND

E-mail address: gorn@mat.uni.torun.pl

PaOlO Nistri

Dipartimento di Ingegneria dell' Informazione

Facoltà di Ingegneria

Università di Siena

Via Roma 56

53100 Siena, ITALY

E-mail address: pnistri@dii.unisi.it

TMNA : Volume $13-1999-\mathrm{N}^{\circ} 1$ 